\title{
Spatial Layout Image Retrieval based on Fast Image Segmentation using K-Means Clustering
}

\author{
D.Binu \\ Assistant Professor \\ Sri Ramakrishna Institute of Technology \\ Coimbatore-641010
}

\author{
P.Malathi \\ Principal \\ Bharathiyar Institute of Engineering for Women \\ Salem-636112
}

\begin{abstract}
An image retrieval system that takes the input query image and retrieves the similar images according to the spatial coordinates and which uses the $\mathrm{k}$ means clustering algorithm for its segmentation. Most existing Content Based Image Retrieval based on the images of color, text documents, informative charts, and shape. This paper aims to search the images with similar spatial layouts and the retrieval process which includes the feature extraction methods and image matching criteria. This paper employs K-Means clustering for image segmentation, and the features of small segments of images are extracted based on the segmentation results. To match the segments of two images, the distance between them can be computed by Eucliedean distance to evaluate the similarity of their spatial layouts.
\end{abstract}

\section{Keywords}

image retrieval, image segmentation, k-means clustering, spatial layout.

\section{INTRODUCTION}

The search by the computation of probabilities very naturally yields an appealing soft query system[1]. This paper provides a solution for image retrieval in mobile devices. Since it might be troublesome for people to manually annotate the photos they take, it is necessary to establish a system which can automatically analyze the content of photos[2].Many of these systems are intended to be used for variable kinds of images on the Internet, and the servers with high performance are usually employed to handle the computation in the image retrieval process. However, the CPU in mobile devices usually has few computational resources, so it is difficult for them to maintain real-time interactions with users. Therefore, a suitable image retrieval system should obtain an acceptable tradeoff between the retrieval performance and the real-time requirement. One of the solutions to the real-time requirement in embedded systems is hardware acceleration, which can effectively speed up the computational speed while maintaining low power consumption. Based on these observations, the proposed work aims at a image retrieval system which integrates a hardware accelerator for mobile devices.

\section{PROPOSED IMAGE RETRIEVAL SYSTEM}

The block diagram of image retrieval system is shown in figure 1 contains the steps involved in the proposed retrieval system. The system gets the image from the data base. Some images are retrieved which is very closer to the query image. The comparison is done by k-means clustering algorithm and Euclidean distance. After that the images are ranked according to the shape, color, texture and spatial co-ordinates. Finally, the images are retrieved according to their ranking.

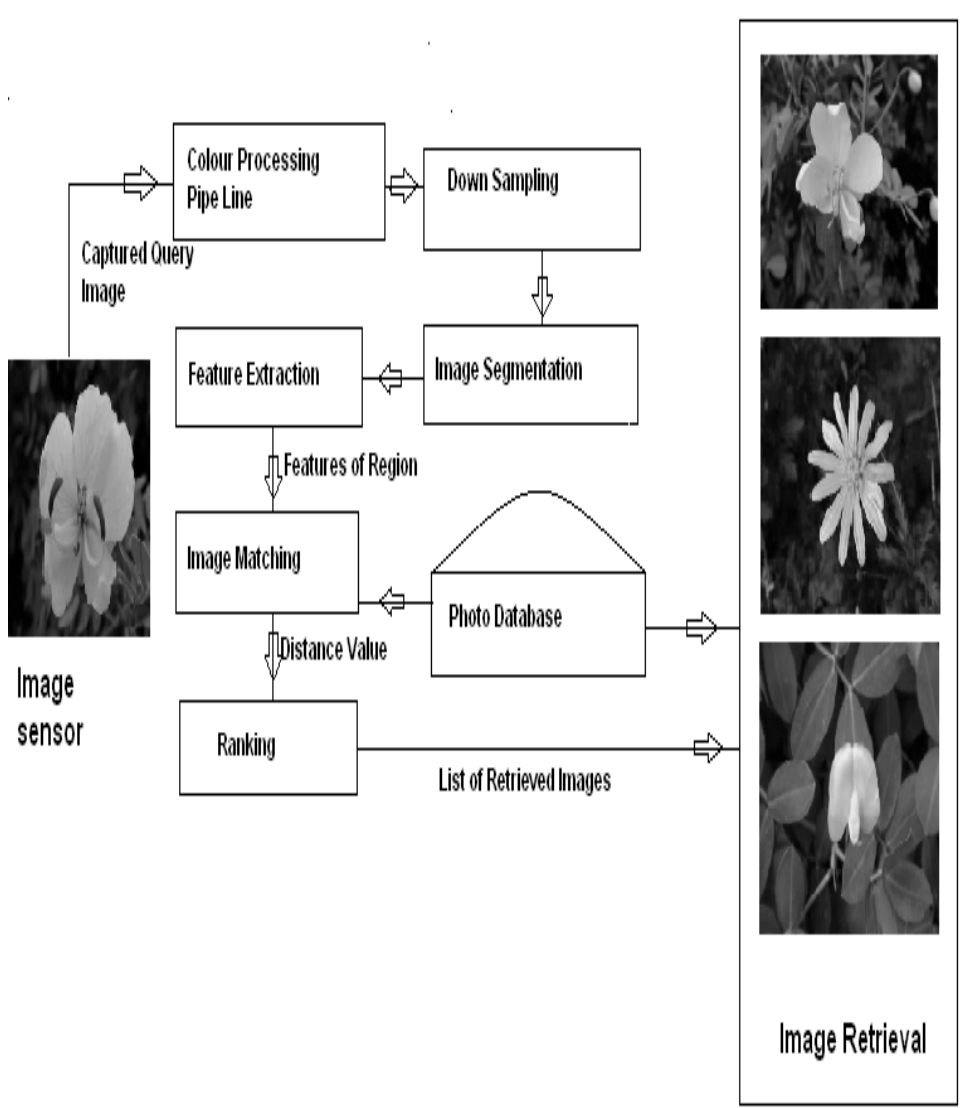

Figure.1 Block diagram of Image retrieval System

\subsection{Preprocessing}

Many color image formats are currently in use, e.g., GIF, JPEG, PPM and TIFF are the most widely used formats. Normalization of the data is important, because images in an image database can have different formats and different sizes. The test data base is relatively small images; a rescaled thumbnail consisting of 128 _ 128 pixels in Red- Green-Blue (RGB) color space is adequate for the purpose of computing the feature vectors.

Since color distances in RGB color space do not reflect the actual human perceptual color distance, convert and store the image in a component color space with intensity and perceived contrasts. We define the new values at a color pixel based on the RGB values of an original pixel as follows: 


$$
\begin{array}{ll}
\mathrm{C} 1 & =(\mathrm{R}+\mathrm{G}+\mathrm{B}) / 3 \\
\mathrm{C} 2 & =(\mathrm{R}+(\mathrm{MAX}-\mathrm{B})) / 2
\end{array}
$$

Here $\max$ is the maximum possible value for each color component in the RGB color space. For a standard 24- bit color image, max. 255. Clearly, each color component in the new color space ranges from 0 to 255 as well. This color space is similar to the opponent color axes

$$
\begin{aligned}
& R G=R-2 * G+B \\
& B Y=-R-G+2 * B \\
& W B=R+G+B
\end{aligned}
$$

Besides the perception correlation properties of such an opponent color space, one important advantage of this alternative space is that the $\mathrm{C} 1$ axis, or the intensity, can be more coarsely sampled than the other two axes on color correlation. This reduces the sensitivity of color matching to a difference in the global brightness of the image, and it reduces the number of bins and subsequent storage in the color histogram indexing.

\section{IMAGE SEGMENTATION}

Color image segmentation is an important step for image processing and analysis. Depending on different applications, various kinds of techniques are used for segmentation. Feature-space methods, such as clustering, intend to classify pixels to different groups in a pre-defined color space, whereas spatial-domain methods, such as region growing, manipulate pixels to form connected regions.

A traditional clustering method, K-Means clustering, is used for image segmentation in the proposed work. K-Means is often employed as an essential step in the segmentation process and it is popular for its simplicity for implementation. The purpose of K-Means is over-segmentation, which decomposes images into small segments, and the decomposed segments indicate spatial regions of images.

There are two reasons using RGB color space. The first is that the feature vector can be directly obtained from the color processing pipelines without applying any color transform. The second is that RGB channels are friendly to hardware design since the bit lengths of three channels in the color space are usually the same, and the data type of pixels is positive integer. To obtain different results of image segmentation, the proposed algorithm can also be performed in other color space, such as $\mathrm{YCbCr}$, YUV, and HSV color space.

\subsection{K-Means clustering}

Cluster analysis or clustering is the assignment of a set of observations into subsets called as clusters so that observations in the same cluster are similar in some sense. Clustering is a method of unsupervised learning, and a common technique for statistical data analysis used in many fields, including machine learning, data mining, pattern recognition, image analysis and bioinformatics[3].

For each cluster is defined as k-centroids. These centroids should be placed in a cunning way because of different location causes different result. So, the better choice is to place them as much as possible far away from each other. The next step is to take each point belonging to a given data set and associate it to the nearest centroid. When no point is pending, the first step is completed and an early groupage is done. At this point we need to re-calculate $\mathrm{k}$ new centroids as barycenters of the clusters resulting from the previous step.
After we have these k new centroids, a new binding has to be done between the same data set points and the nearest new centroid. A loop has been generated. As a result of this loop we may notice that the $\mathrm{k}$ centroids change their location step by step until no more changes are done. In other words centroids do not move any more.

\subsection{K-Means algorithm steps}

The algorithm is composed of the following steps[4]:

Step 1. Place $\mathrm{K}$ points into the space represented by the objects that are being clustered. These points represent initial group centroids.

Step 2. Assign each object to the group that has the closest centroid.

Step 3. When all objects have been assigned, recalculate the positions of the $\mathrm{K}$ centroids

Step 4. Repeat Steps 2 and 3 until the centroids no longer move. This produces a separation of the objects into groups from which the metric to be minimized can be calculated.

A commonly used convergence criterion is

$$
\begin{array}{ccc}
\mathrm{E}=\sum_{\mathrm{Ci}} & \sum|p-m i|^{2} \\
\mathrm{p} \in \mathrm{Ci}
\end{array}
$$

Where mi is the center of cluster $\mathrm{Ci}$.

Figure 2 Shows the flow chart of the K-means Algorithm[5]

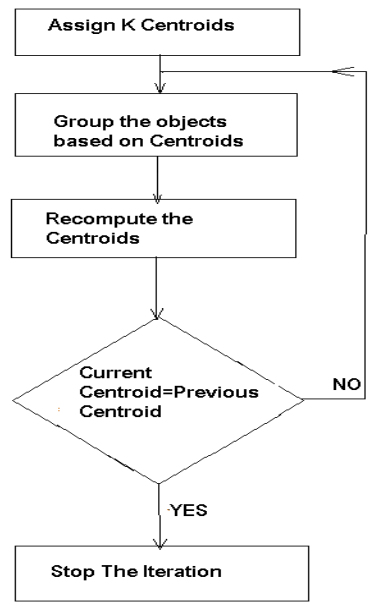

Figure.2 Flow chart of k-means clustering 4. FEATURE EXTRACTION

Feature extraction is defined as locating those pixels in an image that have some distinctive characteristics. Typically that characteristic is some inhomogeneity in local image properties. Step edges, for instance, are inhomogeneities in intensity or range. Range images and amplitude images have different kinds of features that are meaningful; therefore, different approaches are used for each data type. There are two types of features we detect in images. The classical works in edge detection are those of Canny and Marr \& Hildreth.

They perform a fairly comprehensive evaluation and state that due to the research community's success in this area that edge detection as a research topic should die with dignity, as it pertains to step edges in intensity images, but believe that reliable feature detection in range images still poses many questions. In the area of feature extraction in range data, there are also a variety of methods implemented. We divide those methods into four major types. 
A second category is mathematical morphology. In this approach, morphological operators such as erosion, dilation, openings, and closings are used to detect and classify edge types in range data. Edges are detected by the residues obtained from openings and closings with various shaped morphological operators. Krishnapuram and Gupta detect roof edges in range images by identifying the residues rendered by at, one-dimensional structuring element. For a positive roof edge, opening gives a nonzero response, and for a negative roof edge, closing renders a nonzero response.

The third category is multi-scale feature extraction. Multiscale feature extraction used neighborhood operators of various sizes for smoothing or polynomial approximation. In general, the larger the scale, the more the image is smoothed and edges are more likely to be spatially displaced from their actual location in the image. However, the smaller the scale, the more noise remains in the image and interferes with the feature extraction technique.

The fourth category of range image feature extraction is differential geometry. Differential geometry in range images is locating and classifying geometric properties of the scene by means of estimating the derivatives of the digitized range data points and using these estimations to infer the geometry of the surfaces in the range image. Surface curvature, crease edges, and other surface characteristics such as critical points may be obtained through this method. A great deal of work has been done in this area by Besl. In the layout the mathematical methods by which Gaussian and mean curvature may be used to calculate the surface normal of a point in a range image. Maas and Krekel use differential geometry to compute the surface normal to a point in a range image.

\subsection{Color}

Computing distance measures based on color similarity is achieved by computing a color histogram for each image that identifies the proportion of pixels within an image holding specific values. Current research is attempting to segment color proportion by region and by spatial relationship among several color regions. Examining images based on the colors they contain is one of the most widely used techniques because it does not depend on image size or orientation. Color searches will usually involve comparing color histograms, though this is not the only technique in practice.

\subsection{Texture}

Texture measures look for visual patterns in images and how they are spatially defined. Textures are represented by Texel which are then placed into a number of sets, depending on how many textures are detected in the image. These sets not only define the texture, but also where in the image the texture is located. Texture is a difficult concept to represent. The identification of specific textures in an image is achieved primarily by modeling texture as a two-dimensional gray level variation.

\subsection{Shape}

Shape does not refer to the shape of an image but to the shape of a particular region that is being sought out. Shapes will often be determined first applying segmentation or edge detection to an image. Other methods like use shape filters to identify given shapes of an image. In some case accurate shape detection will require human intervention because methods like segmentation are very difficult to completely automate.

\section{IMAGE MATCHING}

The most common method for comparing two images in content based image retrieval is using an image distance measure. An image distance measure compares the similarity of two images in various dimensions such as color, texture, shape, and others. For example a distance of 0 signifies an exact match with the query, with respect to the dimensions that were considered. As one may intuitively gather, a value greater than 0 indicates various degrees of similarities between the images. Search results then can be sorted based on their distance to the queried image.

\section{SIMULATIONRESULTS \&DISCUSSION}

The data base includes pictures of different objects viewed from different angles and scales. The cluster number and segmentation applies different masks as the query image. According to the segmentation and ranking the closely related images are retrieved from the data base.

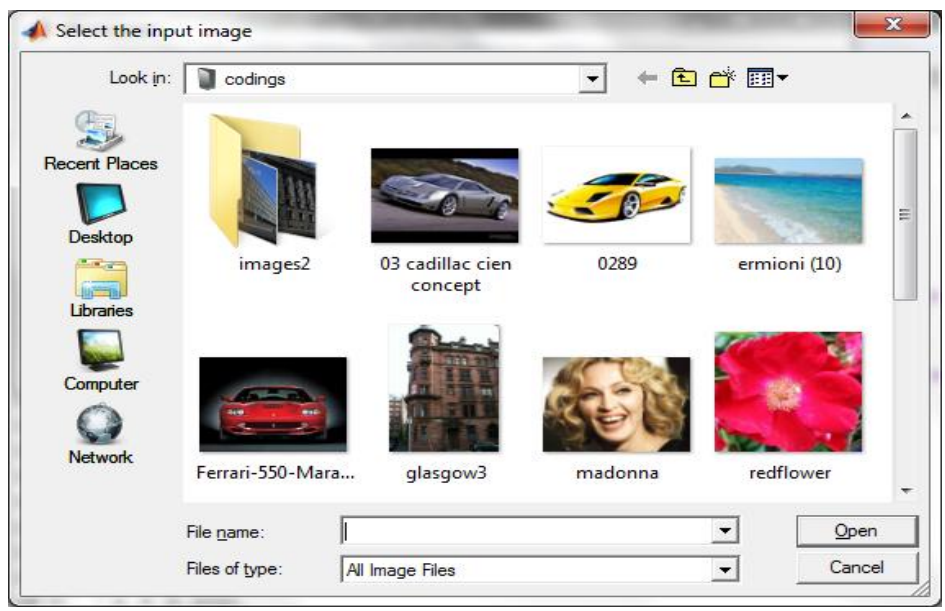

Figure.3 Data base images

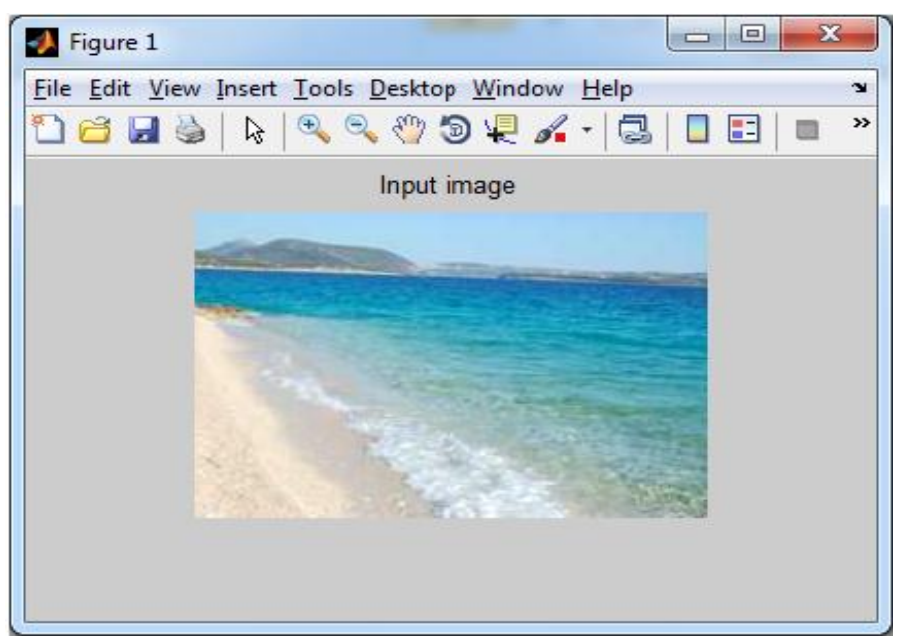

Figure.4 Input image get from user 


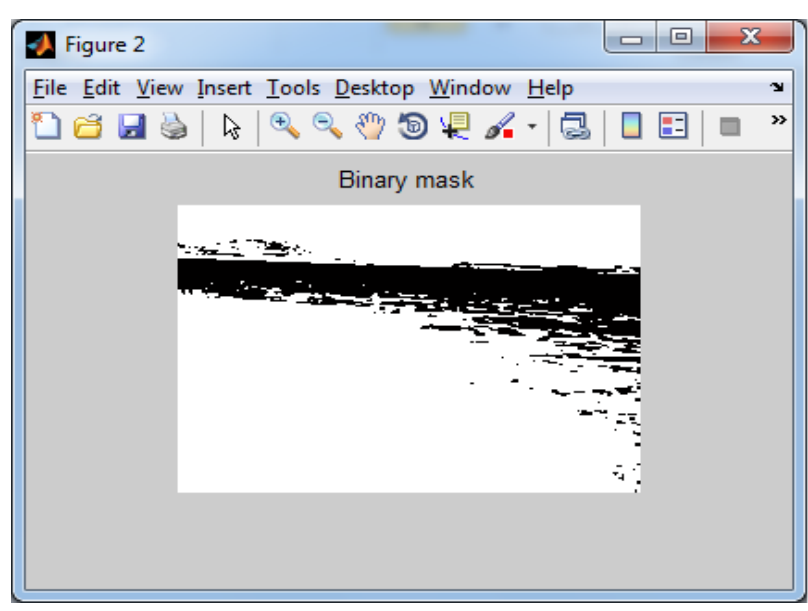

Figure.5 Input image is converted to Binary Image

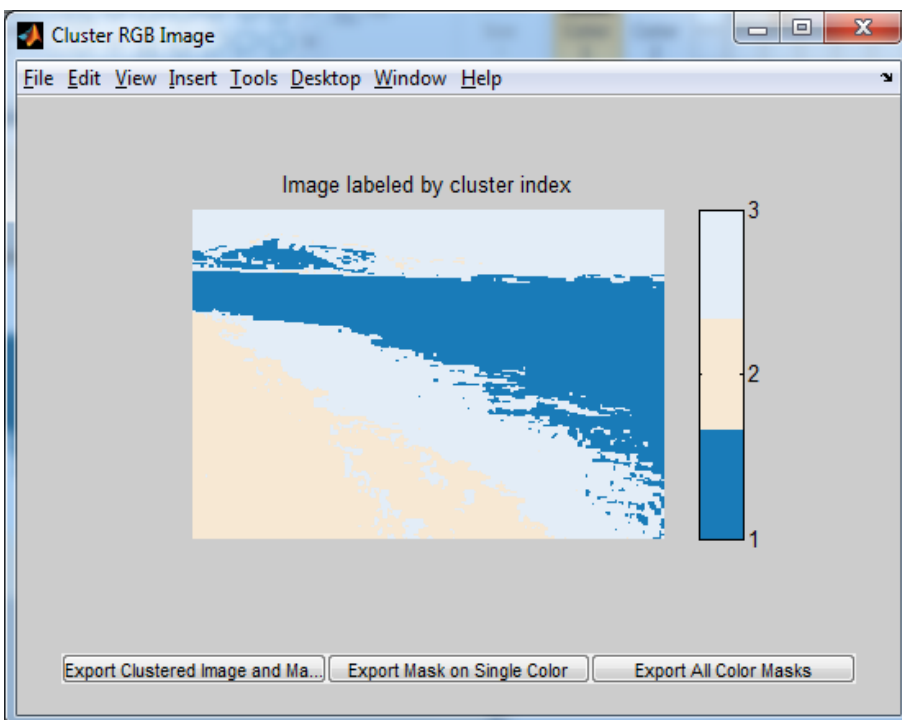

Figure.6 Cluster RGB Image

Figure 1 \begin{tabular}{|l|l|l|}
\hline$口$ & 回 & $\mathbf{x}$ \\
\hline
\end{tabular}

File Edit View Insert Iools Desktop Window Help

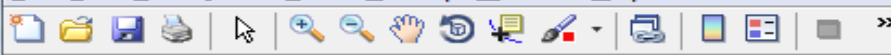

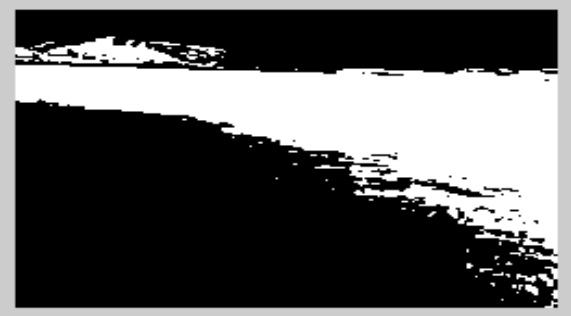

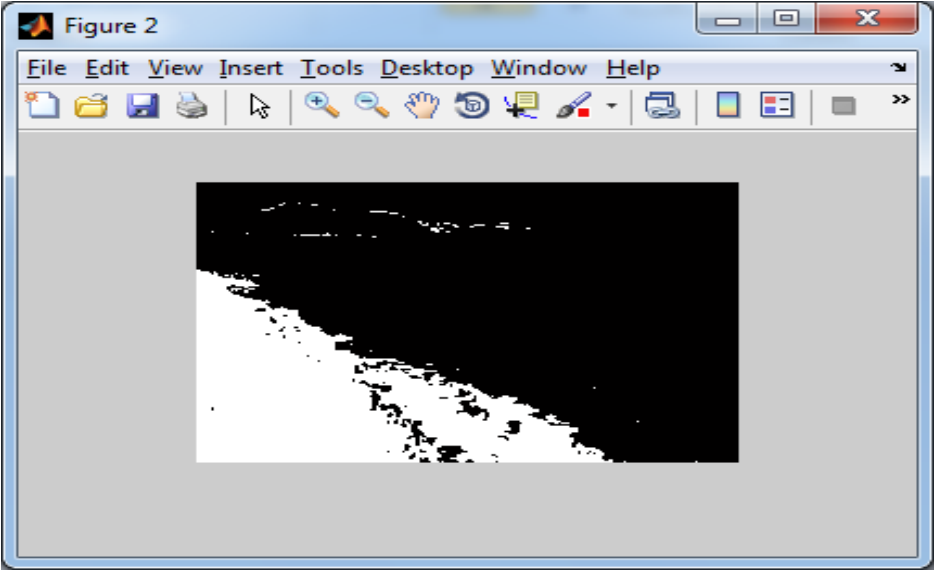

Fig.8 Cluster Mask 2

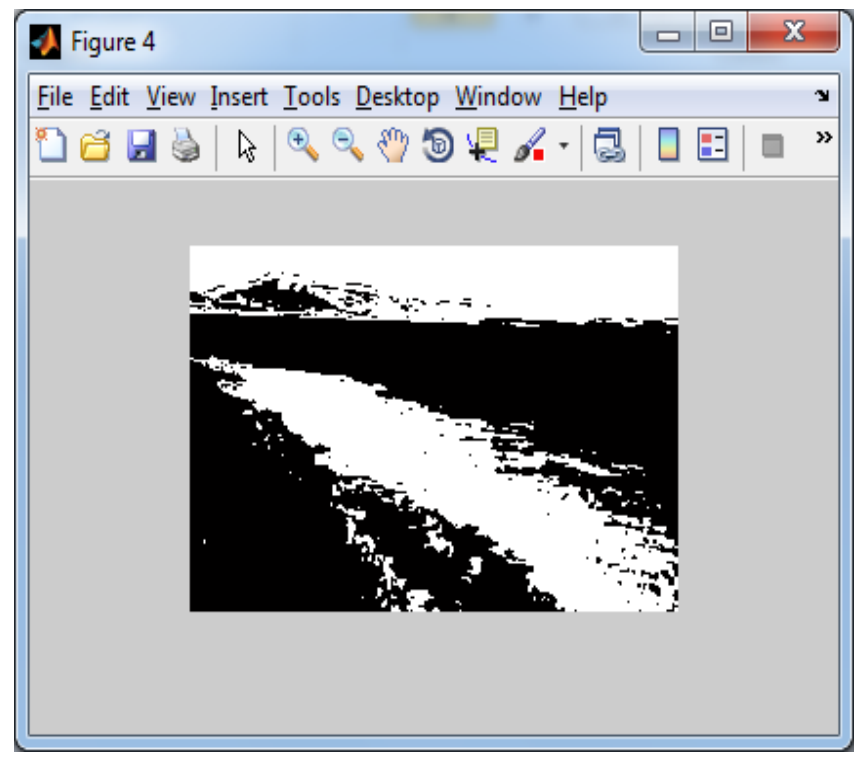

Figure.9 Cluster Mask 3

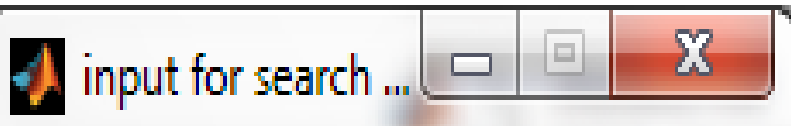

How many image files you want to retrive?

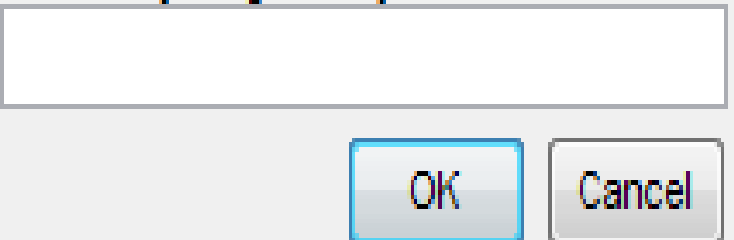

Figure.10 Number of images required

Figure.7 Cluster Mask 1 


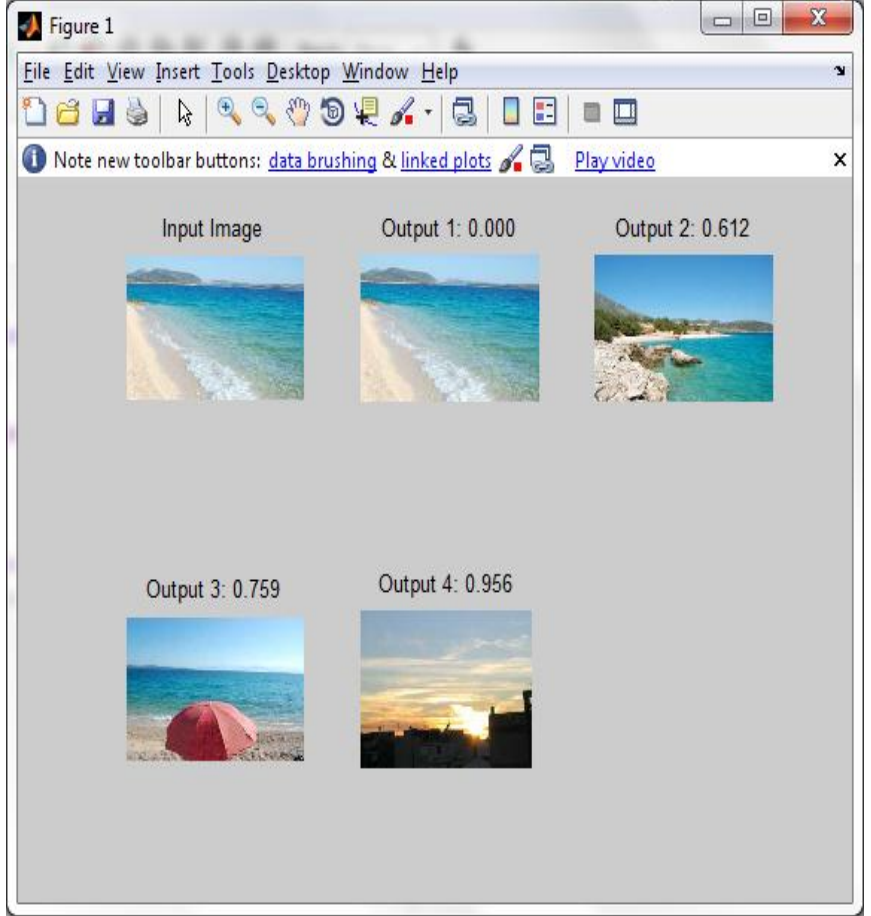

Figure.11 Retrieved images

\section{CONCLUSION AND FUTURE WORK}

This paper presented an approach for Spatial Layout Based Image Retrieval using K-Means clustering techniques where images are initially clustered into groups having similar color content and then the preferred group is clustered using $\mathrm{K}$ Means. K-Means clustering assists faster image retrieval and also allows the search for most relevant images in large image databases. K-Means is a clustering method based on the optimization of an overall measure of clustering quality is known for its efficiency in producing accurate results in image retrieval. Since each cluster obtained is a unique set of similar images, the user can select an image set of his choice and further refine the search by applying K-Means technique. Thus using K-Means techniques together not only facilitates the user not to overlook the image he may require but also to obtain accurate favored image results. For future development the proposed method will be implemented for the real time requirement in hardware.

\section{REFERENCES}

[1] Y. Rui, T. S. Huang, and S.-F. Chang, "Image retrieval: Current techniques, promising directions, and open issues," Journal of Visual Communication and Image Representation, vol. 10, no. 1, pp. 39-62, Mar. 1999.

[2]Tse-Wei Chen,Yi-Ling Chen,and Shao-Yi Chien,'Photo Retrieval Based on Spatial Layout with Hardware Acceleration for Mobile Devices",IEEE Transactoins on Mobile Computing,2011,pp.1-15.

[3] M. Luo, Y.-F. Ma and H.-J. Zhang, "A spatial constrained K-Means approach to image segmentation," in Proceedings of the Joint Conference of International Conference on Information, Communications and Signal Processing, and Pacific Rim Conference on Multimedia, vol. 2, Dec. 2003, pp. 738-742.

[4] T. Kanungo, D. M. Mount, N. S. Netanyahu, C. D. Piatko, R. Silverman, and A. Y. Wu, "An efficient K-Means clustering algorithm: analysis and implementation," IEEE Transactions on Pattern Analysis and Machine Intelligence, vol. 24, no. 7, pp. 881-892, July 2002.

[5] B. K"ovesi, J.-M. Boucher, and S. Saoudib, "Stochastic K-Means algorithm for vector quantization," Pattern Recognition Letters, vol. 22, no. 6, pp. 603-610, 2001. 